\section{Choriocarcinoma with arterial and venous thrombosis}

\section{Sir,}

The treatment of choriocarcinoma forms one of the success stories of oncology with survival rates of nearly $100 \%$ in low-risk group and more than $90 \%$ in the high-risk group. ${ }^{[1]}$ It is a unique neoplasm with a great propensity for spread by hematogenous route, including to the brain. The central nervous system (CNS) metastasis is often hemorrhagic and thormbotic complications are extremely rare.

A 30-year-old lady presented with bleeding per vaginum of two months duration. The obstetric history revealed a normal delivery five years back, a spontaneous abortion four years back, and a molar pregnancy two years back for which evacuation was done. Serum human chorionic gonadotropin (HCG) was 90000 IU. Computerized tomography (CT) of the chest revealed multiple bilateral lung metastasis. CT of the brain was normal.

Based on the history and high HCG values, she was diagnosed to have choriocarcinoma, stage III high risk (WHO score 8), and was initiated on etoposide, methotrexate, actinomycin-D, cyclophosphamide, and vincristine (EMACO) chemotherapy. She responded to treatment with dropping HCG values. After two cycles of the therapy, she had an episode of generalized tonic-clonic seizures. Magnetic resonance imaging (MRI) was suggestive of cerebral venous thrombosis [Figure 1]. Enoxaparin treatment was initiated and was later continued on warfarin. Once her general condition improved EMACO was reinitiated. After two more cycles of the therapy, she had sudden onset right-sided hemiparesis and MRI brain this time was suggestive of left temporal lobe infarct with edema [Figure 2]. Coagulation screening including serum

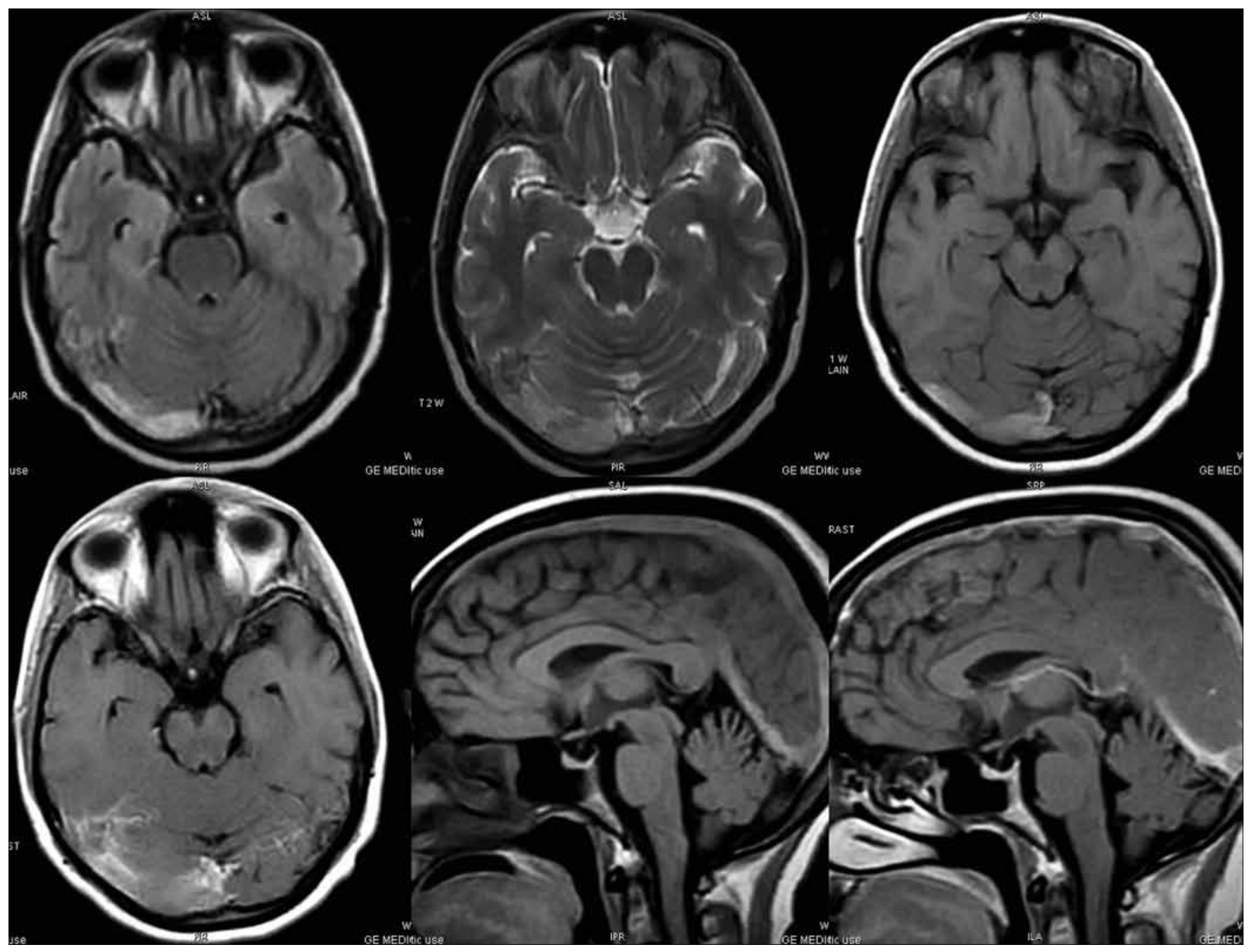

Figure 1: Serial sections showing ill-defined hyperdensity and faint enhancement in the sagittal sinus suggestive of venous thrombosis 

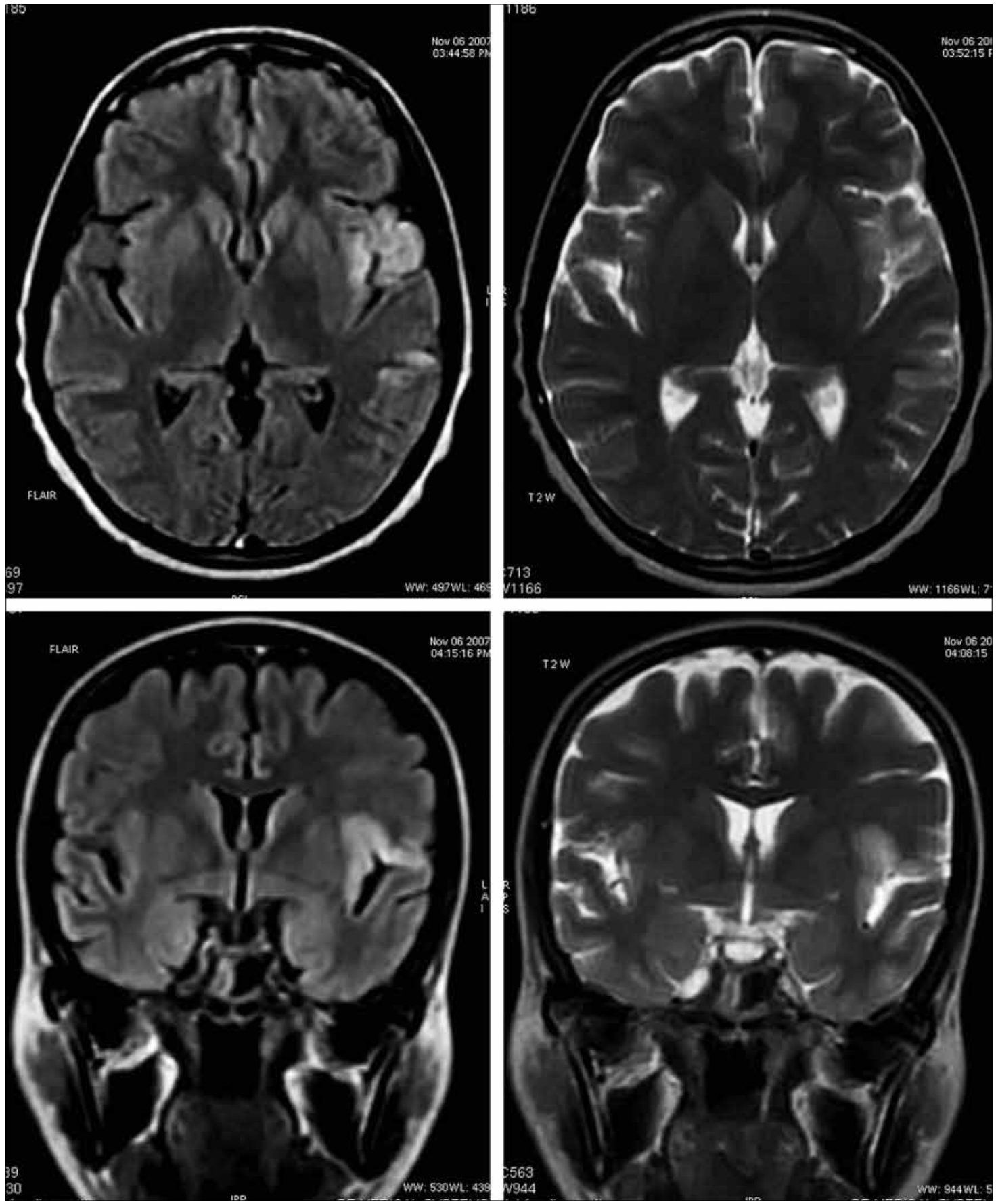

Figure 2: Serial sections showing revasularization of the sagittal venous thrombus with ill-defined altered signals in the temporoparietal cortices

antiphospholipid antibody was normal. Echocardiogram did not reveal any vegetations or septal defects. She was continued on anticoagulants which was stopped only after HCG normalized.

The patient received six cycles of chemotherapy, her HCG values normalized and now she has completed 15 months of follow up. She is in remission and does not have any residual neurological deficits.
Gestation trophoblastic neoplasia represents one of the success stories of modern-era chemotherapy in the treatment of human malignancy. The incidence of choriocarcinoma varies from 1 in 120 pregnancies in some parts of Asia to 1 in 1200 in United States. ${ }^{[1]}$ Choriocarcinoma has the propensity for vascular invasion and widespread metastasis, including to the brain. CNS metastasis is usually hemorrhagic and our patient had normal study of brain upfront and 
developed the neurological events while on treatment. CNS in volvement in Choriocarcinoma can be due to hemorrhagic metastas, tumor embolization and chemotherapy (especially platinum compounds). CNS thrombotic events are extremely rare. The association between cancer and thrombosis was identified in 1865 and the combination is called as Trousseau syndrome. ${ }^{[2]}$ The mechanisms elucidated include cytokines like TNF $\alpha$, IL-1, and IL-6 that cause endothelial damage resulting in thrombosis. ${ }^{[3]}$ The interaction between tumor cells and macrophages activates platelets and factors XII and X, which leads to thrombin generation and thrombosis. The various chemotherapeutic agents including platinum compounds can cause endothelial damage. Central venous catheters and total parenteral nutrition also increase the risk.

Lepidini et al. reported a patient with pure testicular choriocarcinoma who suffered from both arterial and venous thrombosis during chemotherapy. ${ }^{[4]}$ An increased paraneoplastic stimulus of HCG, secondary to the marker surge phenomenon, was suggested as responsible for transient hypercoagulability and subsequent thromboembolism. Komeichi et al. had an interesting case of metastatic choriocarcinoma associated with cerebral thrombosis and aneurysmal formation. ${ }^{[5]}$ The aneurysmal wall was invaded by choriocarcinoma. To the best of our knowledge, this is the first reported case of choriocarcinoma with cerebral venous thrombosis during the course of the treatment. Cerebral thrombotic events also should be borne in mind, when a patient with choriocarcinoma presents with focal deficits.

\section{Sanju Cyriac, T. G. Sagar, Vandana Mahajan}

Cancer Institute (WIA), Chennai, India.

E-mail:drsanpan80@yahoo.com

DOI: $10.4103 / 0028-3886.55586$

\section{References}

1. Muggia FM, Burke TW, Small W Jr. Gestational trophoblastic diseases. In: Devita VT, Hellman S, Rosenberg SA, editors. Cancer. Principles and Practice Of Oncology. $7^{\text {th }}$ ed. Lippincott Williams and Wilkins; 2005. p. 1360-2.

2. Trousseau A. Plegmasia alba dolens: Lectures on clinical medicine, delivered at the Hotel-Dieu, Paris 1865;5:281-32.

3. Bick RL. Cancer associated thrombosis. N Engl J Med 2003;349:109-11.

4. Lepidini G, Biancari F, D'Andrea V. Severe thrombosis after chemotherapy for metastatic choricarcinoma of the testis maintaining complete remission for a long period. Scand J Urol Nephrol 1996;31:221-2.

5. Komeichi T, Igarashi K, Takigami M, Saito K, Isu, Itamo K, et al. A case of metastatic choriocarcinoma associated with cerebral thrombosis and aneurysmal formation. No Shinkei Geka 1996;24:463-7. 